\title{
AJA onlinepublications
}

\section{Summary of Thin-Section Petrographic Analysis Results for Cooking Vessels from Iron Age Philistia}

AN APPENDIX TO "COOKING IDENTITIES: AEGEAN-STYLE COOKING JUGS AND CULTURAL INTERACTION IN IRON AGE PHILISTIA AND NEIGHBORING REGIONS,"BY DAVID BEN-SHLOMO, ITZHAQ SHAI, ALEXANDER ZUKERMAN, AND AREN M. MAEIR (AJA 112 [2008] 225-46).

\begin{tabular}{|c|c|c|c|c|c|c|c|}
\hline Sample & Type & Site & Stratum & Reg. No. & Publication & $\begin{array}{l}\text { Petrographic } \\
\text { Group }^{\mathrm{a}}\end{array}$ & Soil Type \\
\hline P1 & 2 & Miqne & VIIB & IVNW.41.222.5 & - & 1 & brown \\
\hline P2 & 2 & Miqne & VIIA & IVNW.41.215.1 & - & 1 & brown \\
\hline P3 & 2 & Miqne & VIIA & IVNW.41.217.3 & - & 2 & brown/loess \\
\hline P4 & 2 & Miqne & VIIA & IVNW.41.246.16 & - & 2 & loess \\
\hline P5 & 2 & Miqne & VIIA & IVNW.24.377.15 & - & 2 & brown/loess \\
\hline P6 & 2 & Miqne & VIIA & IVNW.42.348.8 & - & 1 & brown \\
\hline P7 & 2 & Miqne & VIIB & IVNW.43.495.5 & - & 1 & brown \\
\hline P8 & 2 & Miqne & VIIA & IVNW.25.291.1 & - & 1 & brown \\
\hline P9 & 2 & Miqne & VIA & IVNE.23.328.1 & - & 1 & brown \\
\hline P10 & 2 & Miqne & VB & IVNE.9.163.19 & - & $1 b$ & brown \\
\hline P11 & 2 & Miqne & VB & IVNW.9.383.3 & - & 2 & $\begin{array}{c}\text { brown/loess?; } \\
\text { quartz sand added }\end{array}$ \\
\hline P12 & 2 & Miqne & VB & IVNW.27.226.30 & - & 2 & loess/calcareous \\
\hline P13 & 2 & Miqne & VC & IVNE.24.331.4 & - & 2 & loess \\
\hline P14 & 2 & Miqne & VC & IVNE.23.293.11 & - & $1 b$ & brown/loess \\
\hline P15 & 2 & Miqne & VIA & IVNW.39.128.1 & - & $1 \mathrm{a}$ & $\begin{array}{c}\text { brown; } \\
\text { quartz sand added }\end{array}$ \\
\hline P16 & 1 & Miqne & VIB & IVNW.24.331.7 & - & $3 a$ & loess/calcareous \\
\hline P17 & 1 & Miqne & VIA & IVNW.8.316.5 & - & $3 b$ & calcareous marl \\
\hline P18 & 1 & Miqne & VB & IVNW.7.71.2 & - & $3 a$ & loess/calcareous \\
\hline P19 & 1 & Miqne & VB & IVNW.7.71.4 & - & $3 a$ & loess/calcareous \\
\hline P20 & 4 & Miqne & IVA & IVNW.25.155.7 & - & 1 & brown \\
\hline $\mathrm{P} 21$ & 2 & Ashdod & XII & $\mathrm{H} 1374 / 2$ & $\begin{array}{l}\text { Dothan and Ben-Shlomo } \\
\text { 2005, fig. } 3.28, \text { no. } 7\end{array}$ & $1 \mathrm{a}$ & brown \\
\hline P22 & 2 & Ashdod & XII & $\mathrm{H} 1304 / \mathrm{C}$ & $\begin{array}{l}\text { Dothan and Ben-Shlomo } \\
2005 \text {, fig. } 3.28 \text {, no. } 10\end{array}$ & $1 c$ & brown (hamra?) \\
\hline P23 & 2 & Ashdod & XII & H1382 & $\begin{array}{l}\text { Dothan and Ben-Shlomo } \\
\text { 2005, fig. } 3.28 \text {, no. } 11\end{array}$ & $1 \mathrm{a}$ & brown \\
\hline P24 & 2 & Ashdod & Xla & H3268 & $\begin{array}{l}\text { Dothan and Ben-Shlomo } \\
\text { 2005, fig. } 3.58 \text {, no. } 6\end{array}$ & $1 a$ & brown \\
\hline P25 & 2 & Ashdod & $\mathrm{XI}$ & $\mathrm{H} 758 / 1$ & $\begin{array}{c}\text { Dothan and Ben-Shlomo } \\
2005 \text {, fig. } 3.58, \text { no. } 13\end{array}$ & $1 \mathrm{a}$ & brown \\
\hline P26 & 2 & Ashdod & $X I$ & $\mathrm{H} 1759$ & $\begin{array}{c}\text { Dothan and Ben-Shlomo } \\
\text { 2005, fig. } 3.58, \text { no. } 8\end{array}$ & $2 a$ & loess \\
\hline
\end{tabular}




\begin{tabular}{|c|c|c|c|c|c|c|c|}
\hline Sample & Type & Site & Stratum & Reg. No. & Publication & $\begin{array}{l}\text { Petrographic } \\
\text { Group }\end{array}$ & Soil Type \\
\hline SF74 & 1 & Safi & A5-A4 & $630133 / 1$ & - & $3 a$ & loess \\
\hline SF75 & 1 & Safi & A5-A4 & $330275 / 1$ & - & $3 a$ & loess \\
\hline SF76 & 3 & Safi & A5-A4 & $630010 / 2$ & - & $3 a$ & loess \\
\hline SF77 & 3 & Safi & A5-A4 & $530072 / 15$ & - & $3 b$ & brown \\
\hline SF78 & 3 & Safi & A5-A4 & $530050 / 1$ & - & $3 a$ & loess \\
\hline SF79 & 3 & Safi & A5-A4 & $530091 / 39$ & - & $3 b$ & brown \\
\hline SF80 & 3 & Safi & A5-A4 & $530056 / 8$ & - & $3 a$ & brown \\
\hline SF81 & 2 & Safi & A5-A4 & $530255 / 6$ & - & $2 a$ & loess \\
\hline SF82 & 2 & Safi & A5-A4 & $730307 / 8$ & - & $2 d$ & loess \\
\hline SF83 & 2 & Safi & A5-A4 & $730045 / 4$ & - & $1 \mathrm{~d} ?$ & brown? \\
\hline SF84 & 2 & Safi & A5-A4 & $230044 / 15$ & - & $2 b$ & loess \\
\hline SF100 & 2 & Safi & A5-A4 & $670163 / 17$ & - & $2 a$ & loess \\
\hline SF101 & 2 & Safi & A5-A4 & $670157 / 5$ & - & $3 a$ & loess \\
\hline SF102 & 2 & Safi & A5-A4 & 450265 & - & $1 \mathrm{a}$ & brown \\
\hline SF106 & 5 & Safi & $\mathrm{A} 3$ & $450216 / 2$ & - & $1 b$ & brown \\
\hline SF107 & 5 & Safi & $\mathrm{A} 3$ & $230311 / 4$ & - & $1 b$ & brown \\
\hline SF108 & 5 & Safi & $\mathrm{A} 3$ & $220245 / 1$ & - & $1 b$ & brown \\
\hline SF109 & 5 & Safi & $\mathrm{A} 3$ & $450355 / 2$ & - & $1 \mathrm{a}$ & brown \\
\hline SF110 & 5 & Safi & $\mathrm{A} 3$ & $230363 / 19$ & - & $1 b$ & brown \\
\hline SF111 & 5 & Safi & $\mathrm{A} 3$ & $220242 / 1$ & - & $1 d$ & $\begin{array}{c}\text { brown; } \\
\text { quartz sand added }\end{array}$ \\
\hline SF112 & 5 & Safi & $\mathrm{A} 3$ & $420143 / 2$ & - & $1 b$ & brown \\
\hline SF113 & 4 & Safi & $\mathrm{A} 3$ & 450182 & - & $2 b$ & loess \\
\hline SF114 & 5 & Safi & $\mathrm{A} 3$ & $220329 / 2$ & - & $2 b$ & loess \\
\hline SF115 & 5 & Safi & A3 & $220232 / 1$ & - & $1 b$ & brown \\
\hline SF116 & 5 & Safi & $\mathrm{A} 3$ & $120108 / 1$ & - & $1 \mathrm{a}$ & brown \\
\hline SF117 & 5 & Safi & A3 & $230179 / 8$ & - & $1 a$ & brown \\
\hline SF138 & 1 & Safi & E4 & $560187 / 2$ & - & $3 a$ & loess \\
\hline
\end{tabular}

${ }^{a}$ For details, see Ben-Shlomo 2006, 165-79

\section{Works Cited}

Ben-Shlomo, D. 2006. Decorated Philistine Pottery: An Archaeological and Archaeometric Study. BAR-IS 1541. Oxford: Archaeopress.

Dothan, M., and D. Ben-Shlomo. 2005. Ashdod VI: Excavations of Areas H and K (1968-1968). Israel Antiquities Authority Reports 24. Jerusalem: Israel Antiquities Authority. 